\title{
EXPERIMENTAL EVALUATION OF SELECTED METHODS FOR MULTIGROUP TRAIN FORMATION
}

The purpose of this research project was to compare advantages and disadvantages of selected methods for multigroup train formation. Three simulation models of the marshalling yards were developed to generate and evaluate results for the selected methods. This paper summarizes the steps of the analysis and the results.

Key words: multigroup train formation, marshalling yard, computer simulation

\section{Introduction}

Railway transport influences the growth of many national economies. The railway transport should improve its competitiveness against other modes of transport, in particular the road transport. The following measures have to be taken: 1) implementation of research results and modern control methods and better utilization of the system capacity; 2) modernization of its infrastructure and equipment; 3) cost cutting through employment reduction; 4) improvement of the energy management and consumption; 5) safety improvements; and, 6) implementation of information technologies.

Train formation is one of key elements influencing the effectiveness and profitability of the freight railway transport. The process of train formation is costly and time consuming, requires significant resources, and produces a relatively high number of workrelated injuries.

Presently, two key trends influence the profitability of the train formation process:

- consolidation of the train formation process in a number of highly-specialized and well-equipped marshalling yards, and

- increased customer demand for timely deliveries to support their just-in-time production.

Solutions to address these trends can be organized in the following three categories:

- operation management,

- infrastructure, and

- information systems.

This paper focuses on analyzing potential solutions within the operation management category. In particular, we examine the selection of the most effective method for multigroup train formation.

\section{Methods for multigroup train formation}

In general, the methods for multigroup train formation can be classified in two main groups [1]:
- step methods, and

- simultaneous methods.

Under the step method, the cars are first sorted based on the direction of their destination and assembled randomly on sorting tracks. Next, the cars are assembled sequentially into their final train formations based on the predetermined groups. In the second step, two activities are performed:

- move and arrange individual cars to sorting tracks, and

- assemble sorted cars into final trains.

Under the simultaneous method, the cars are first sorted to groups and then, in the second step, sorted based on the direction of their destination. Therefore, at the end of sorting, all cars are assembled in the final trains.

\section{Research methodology}

At the beginning of this research, we chose parameters whose values were used to evaluate and compare the selected methods for multigroup train formations. The term "Parameter Value" refers to values derived from measured characteristics, which can be used as a criterion for evaluating and comparing the selected methods.

Methods were evaluated and compared using the following criteria:

- time required for formation of a selected number of trains,

- number of tracks used for sorting,

- number of backward movements of humping locomotive to resort cars,

- number of movements of shunting locomotive to assemble outbound trains,

- time of use of the humping track,

- distance traveled by the humping locomotive, and

- distance traveled by the shunting locomotive.

\footnotetext{
* Peter Márton

University of Žilina, Faculty of Management Science \& Informatics, Tel. +421 41 5134229, E-mail: Peter.Marton@fri.utc.sk
} 
Both types of methods, step methods (traditional, square, simple, double, imposition, balanced increase) [1] and simultaneous methods (elementary, triangular, geometric, geometric with backward sorting) [2], [3], [4] were analyzed.

Specific methods of each type were selected based upon their actual and potential use.

The purpose of this analysis was to:

1. conclude whether it is possible to establish empirically mathematical formulas that could be used, given specific inputs (i.e., number of trains), to estimate time needed for a certain number of multigroup trains formation and, at the same time, evaluate potential use of these mathematical relationships for general use in various applications,

2. conclude whether it is possible to find some mutual dependences among formulas, described in point 1 above,

3. conclude whether it is possible to observe a statistically significant correlation between time required for train formation and all other selected parameters, and

4. formulate general recommendations for multigroup train formation in practice.

\subsection{Computer simulation}

Parameter Values were derived from a computer simulation.

The term "computer simulation" should be understood as an experimental analysis of real-life processes using a computer simulation model. The use of a simulation model enables performing a large number of experiments, generating and evaluating outputs, and identifying the optimal solution for use in the real-life application.

\subsection{Villon simulation tool}

Simulation models used for estimation of Parameter Values were built using the Villon simulation tool. This tool was developed in cooperation between the University of Žilina and the Simcon company. This tool is used for computer simulation of railway operation as well as operation of other transport modes.

The Villon simulation tool allows simulation results to be recorded to output files. Data recorded to the output files may be presented as a table, diagram or figure. These data may then be exported in an Excel format for further analysis and evaluation [5].

\subsection{Simulation models used for examination of methods of multigroup train formation}

Three simulation models of marshalling yards were created to generate Parameter Values:

- VirBa,

- Stará Harfa,

- Teplička.
The VirBa simulation model was built at first. The infrastructure of VirBa model has the serial layout of siding groups. The infrastructure used in this model is fictitious. Other two models Stará Harfa model and Teplička model - followed thereafter. These models were built to verify VirBa model results. Stará Harfa model uses infrastructure of Žilina zr.st. marshalling yard. This infrastructure was chosen in particular because of this yard's parallel layout of siding groups. Teplička model uses infrastructure of ŽilinaTeplička marshalling yard, which is presently under construction. This infrastructure was chosen in particular because of the yard's serial layout of siding groups that is the only one of the kind in the Slovak Republic.

\subsection{Simulation experiments}

Each simulation model was built to run a specific number of experiments. These experiments were identical for each of the three models. As mentioned earlier, each model was used to analyze the ten selected methods. For each method, six individual experiments were performed. These six experiments compose one test group and differ from each other by a number of assembled outbound trains.

The first experiment begins with two trains and the number of the trains increases by one in the subsequent experiments to a final number of seven outbound trains in the sixth experiment.

Assembled outbound trains are formed by cars that enter the simulation models on inbound trains.

The number of inbound trains and assembled outbound trains is equal in each experiment (i.e. all the cars that enter the simulation model on inbound trains exit the simulation model in assembled outbound trains).

Each train is composed of the same number of cars, namely nine cars. A unique destination is defined for each car. It means that during the secondary sorting each car belongs to a different group. The car (group) order in the inbound trains is generated randomly from a uniform distribution with parameters 1 and 9 .

In each simulation model, ten packets of the simulation experiments were designed (i.e. one observed method $=$ one packet). . Each packet consists of six simulation experiments. So for each simulation model 60 simulation experiments were defined.

\section{Research results}

To answer the questions discussed in part 3 (the purpose of analysis), it was necessary to analyze Parameter Values obtained from computer simulation.

The analysis consisted of the following steps:

1. to evaluate whether formation time of outbound trains correlates to a number of assembled outbound trains and to derive correlation formulas $(y=a x+b)$,

2. to analyze correlation coefficients $(a, b)$ from formulas described in point 1 above and the correlation between calculated coefficients, 
3. to examine whether (and to what degree) Parameter Values affect time needed for train formation, and

4. to compare results among the simulation models.

\subsection{Correlation between time needed for train formation and a number of assembled outbound trains}

In this step, data consisting of the start time of the departure sidings occupation were analyzed to examine the correlation between time needed for train formation and a number of assembled outbound trains. The occupation starts after displacement of correctly assembled outbound trains.

From the measured Parameter Values we derived the following formula for each method. This formula indicates linear dependence between the formation time and a number of assembled outbound trains.

\subsection{Analysis of correlation coefficients $(a, b)$ and the correlation between these coefficients}

The objective was to analyze differences and ratios among correlation coefficients derived from the selected methods. Results were compared across simulation models.

Results (differences and ratios) obtained from simulation models were not equal. Therefore, it is not possible to generalize results of any of the simulation models. In other words, results from the simulation of one marshalling yard can not be used in another marshalling yard.

\subsection{Influence of Parameter Values to time needed for train formation}

In this step, we evaluated whether linear dependence exists between selected Parameter Values. The following pairs of selected Parameter Values were analyzed:

- distance traveled by the humping locomotive (independent value) and formation time required for a selected number of trains (dependent value),

- distance traveled by the shunting locomotive and the formation time required for a selected number of trains,

- distance traveled by the humping locomotive and the time of use of the humping track,

- number of backward movements of humping locomotive to resorting cars and the distance traveled by the humping locomotive,

- number of movements of shunting locomotive to assemble sorted cars into final train and the distance traveled by the shunting locomotive,

- number of tracks used for sorting and the time required to assemble a selected number of trains from ordered groups from sorting sidings

- number of tracks used for sorting and the number of backward movements of humping locomotive to re-sorting cars,
- number of tracks used for sorting and the number of movements of shunting locomotive to assemble sorted cars into final train.

In addition, ratios among all Parameter Values were analyzed. The purpose of this calculation was to find out if the ratio of values of two selected Parameter Values of the selected method is equal for each number of assembled outbound trains. If a ratio of a pair of Parameter Values is equal for a different number of assembled outbound trains, then it is possible to declare the validity of this ratio for the specific marshalling yard. In this case it would be sufficient to calculate the ratio between selected Parameter Values only for one number of assembled outbound trains. Following this it may be possible to calculate another Parameter Value for the remaining number of assembled outbound trains. It will be not necessary to obtain this Parameter Value with the help of computer simulation.

The results of this analysis did not fulfill the expected assumptions. Therefore, it is not possible to generalize the results from one simulation model and use the results from one marshalling yard in another marshalling yard.

The ratio analysis for selected Parameter Values showed that only the simulation models with serial layout of siding groups produce approximately equal results for each observed number of assembled trains.

\subsection{Comparison of results among simulation models}

Finally, the results of the simulation analysis were used to develop ranking of the observed methods for multigroup train formation. Criteria of ranking were all Parameter Values.

By comparison based on formation time required for three or more assembled outbound trains, the three best methods were triangular simultaneous method, geometric simultaneous method and geometric simultaneous method with backward sorting. Only by formation of two trains the traditional step method was the most efficient.

The traditional step method showed the best results by comparison based on distance traveled by the humping locomotive by assembling two or three trains. Only in the Stará Harfa model the traditional step method was the best method in the formation of four trains. By formation of two, three or four trains (the Stará Harfa model) these methods follow in rank - triangular simultaneous method, geometrical simultaneous method and geometrical simultaneous method with backward sorting.

The simultaneous methods achieved the best results by comparison based on distance traveled by the shunting locomotive because after the secondary sorting, the cars on the outbound train are correctly assembled. As a result, the distance traveled by the shunting locomotive equals zero. When step methods are used, it is necessary to assemble cars to train and to move them to the departing group. The traditional step method showed the worst result at comparison based on this criterion. Other step methods have approximately equal results (values are about $75 \%$ of the value measured for the traditional step method). 
The top three methods based on time of use of the humping track for three to seven assembled outbound trains were respectively - the triangular simultaneous method, geometric simultaneous method and geometric simultaneous method with backward sorting. The traditional step method was the best method only when forming two trains.

When comparing the number of tracks used for sorting, the geometric simultaneous method and the geometric simultaneous method with backward sorting were equally effective and were the most efficient. The following methods ranked in efficiency as follows - triangular simultaneous method, square step method, imposition step method and double step method. The elementary simultaneous method and the traditional step method showed the worst results.

By formation of two or three trains the traditional step method showed the best results by comparison based on the number of backward movements of the humping locomotive in order to re-sort cars. In the formation of four trains, the traditional step method and all simultaneous methods (except the elementary simultaneous method) showed equal results. By the formation of five or more trains the simultaneous methods (except elementary simultaneous method) showed the top three results.

The simultaneous methods were the most efficient based on the number of movements of the shunting locomotive in order to assemble the sorted cars into the final train, because they are correctly assembled on the outbound trains after the secondary sorting is finished. These methods are followed by the imposition step method. The traditional step method was the least efficient.

\section{Conclusion}

The following facts (in reference to the research goals from part 3) were found by the experimental evaluation of selected methods for multigroup train formation:

1. It is possible to estimate the formula from the measured Parameter Values for dependence between formation time and a number of assembled outbound trains. This formula signifies the linear dependence of time on the number of trains (Fig. 1). The comparison of coefficients indicated that it is not possible to generalize the results of any of the simulation models. Therefore, we conclude that it is not possible to derive generally valid formula to calculate time required for train formation in any marshalling yard.

2. The differences and ratios of correlation coefficients $(a, b)$ of linear dependence formulas obtained from simulation models were not equal. Therefore, we conclude that it is not possible to generalize the observation results from any of the simulation models.

3. The analysis of ratios between the selected Parameter Values showed that only the simulation models with a serial layout of siding groups produce approximately equal results for each observed number of assembled outbound trains.

4. The triangular simultaneous method, geometric simultaneous method and simultaneous geometric method with backward sorting produced the best results using any of the Parameter Values (Fig. 1)

Acknowledgment

This paper was supported by the Slovak grant foundation under grant $1 / 1049 / 04$

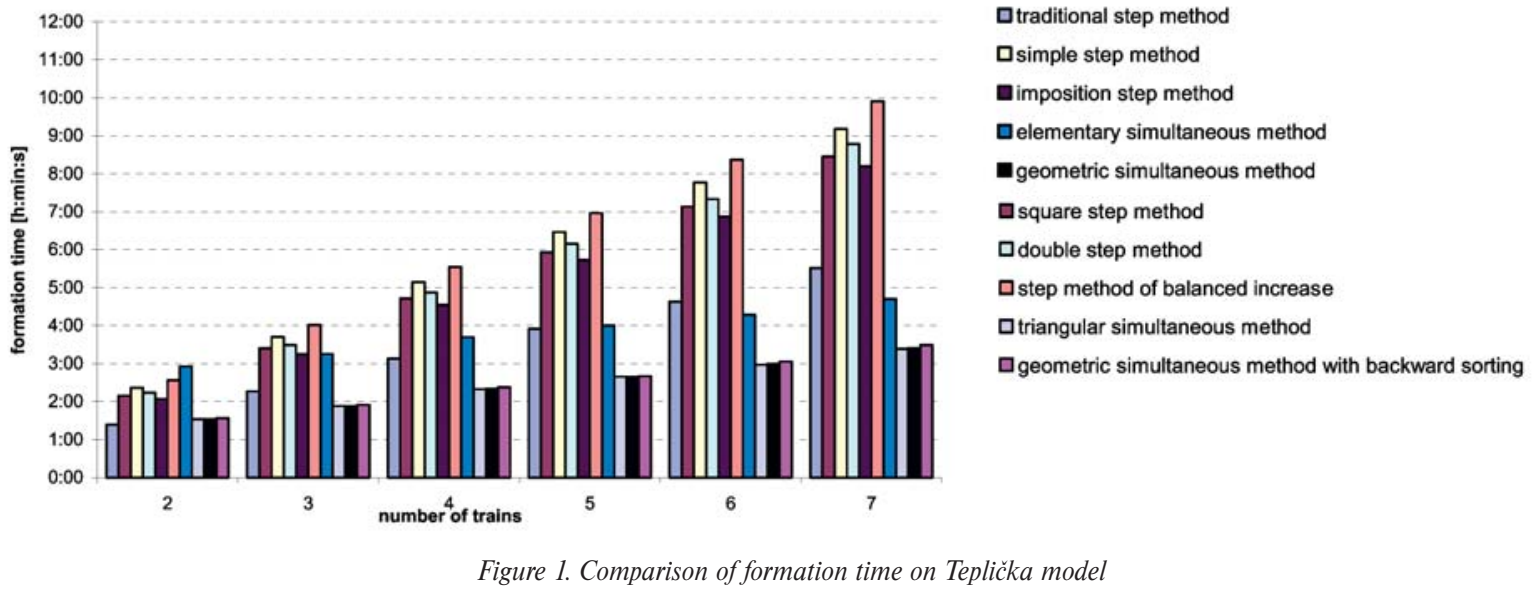

\section{References}

[1] KECLÍK, J.: Mechanics in Railway Operation, Alfa, Bratislava 1978

[2] KRELL, K.: Grundgedanken des Simultanverfahrens, Rangiertechnik Nr. 22, Hersta-Verlag, Hamburg, 1962, pp. 15-23.

[3] KRELL, K.: Ein Beitrag zur gemeinsamen Bildung von Nahgüterzügen, Rangiertechnik Nr. 23, Hersta-Verlag, Hamburg, 1963, pp. 16-25.

[4] PENTIGMA, K.: Teaching Simultaneous Marshalling, The Railway Gazette, Sutton Surey, 1959, pp. 590-593.

[5] TALKE, W.: Effizienzsteigerung in Zugbildungsanlagen, Eisenbahntechnische Rundschau Nr. 11 \& Nr. 12, Hersta-Verlag, Hamburg, 1998, pp. 691-695, 768-775. 